of orthogonal functions, leads, if applied to the case $f=g$ to Bessel's identity

(6) $\int_{R}\left[f-\sum_{p=1}^{n} \phi_{p} \int_{R} f \phi_{p} d R\right]^{2} d R=\int_{R} f^{2} d R-\sum_{p=1}^{n}\left[\int_{R} f \phi_{p} d R\right]^{2}$,

from which Bessel's inequality immediately follows.

5. Theorems analogous to those of the present note involving finite sums or infinite series in place of integrals may be proved in a similar manner.

GöTTINGKN,

June, 1909.

\title{
ON THE TACTICAL PROBLEM OF STEINER.
}

BY PROFESSOR W. H. BUSSEY.

(Read before the American Mathematical Society, February 24, 1906.)

THE study of tactical configurations known as triple systems had its origin in two problems proposed independently by J. Steiner $*$ and T. P. Kirkman. $\dagger$ The Steiner problem, which is the more general and includes the other, is as follows:

For what values of $n$ is it possible to arrange $n$ elements in sets of three, called triads, so that every set of two elements is contained in one and only one triad? If $n$ is a number for which there is such an arrangement in triads, are there other arrangements that cannot be obtained from it by a mere permutation of the elements? When such an arrangement in triads has been made, is it possible to arrange the $n$ elements in sets of four, called tetrads, so that no triad is contained in a tetrad and so that every set of three that is not a triad is contained in one and only one tetrad? When such an arrangement in tetrads has been made, is it possible to arrange the $n$ elements in sets of five, called pentads, so that no triad or tetrad is contained in a pentad, and so that every set of four that is not a tetrad and does not contain a triad is contained in one and only one pentad? In general, when an arrangement in $k-a d s$ has been made, is it possible to arrange the $n$ elements in sets of $k+1$, called $(k+1)-a d s$ so that no $l-a d(l \leqq k)$ is contained in a $(k+1)-a d$, and so that every set of $k$ elements that is not a

\footnotetext{
* Journal für die reine und angewandte Mathematik, vol. 45, p. 181.

†The Lady's and Gentleman's Diary for 1850. For other references to the literature of Kirkman's fifteen school girls problem see Ball's Mathematical Recreations and Essays, 4th edition, page 121.
} 
$k$-ad and does not contain an $l-a d(l<k)$ is contained in one and only one $(k+1)$-ad?

The part of the problem that relates to triads has been completely solved. * The other parts have been little studied.

If an arrangement of $n$ elements in triads, tetrads, pentads, etc., is possible, the number of $k$-ads for $k=3,4,5, \ldots$ is given by the formula

$$
N_{k}=\frac{1}{k !} n(n-1)(n-3) \cdots\left(n-\left[2^{k-2}-1\right]\right) .
$$

This formula was suggested by Steiner. It may be proved without much difficulty by complete induction.

This paper has to do with the case in which $n$ is a number of the form $2^{j}-1$. Its object is to show that it is possible to arrange such a number of elements in $k$-ads for $k=3,4,5$, $\cdots, j+1$. The formula gives $N_{k}=0$ when $k>j+1$.

Consider the $2^{k+1}-1$ elements $\left(x_{1}, x_{2}, x_{3}, \cdots, x_{k+1}\right)$, each $x$ being 0 or 1 and the element $(0,0,0, \ldots, 0)$ being excluded. For convenience the language of geometry is used and each of the elements is called a point. The $2^{k+1}-1$ points are said to constitute a finite geometry of $k$ dimensions, or, more briefly, a $k$-space. $\dagger$ Consider also the linear homogeneous congruence, modulo 2 ,

$$
a_{1} x_{1}+a_{2} x_{2}+a_{3} x_{3}+\cdots+a_{k+1} x_{k+1} \equiv 0,
$$

in which each coefficient is 0 or 1 and at least one of them is not zero. The points of the $k$-space that satisfy such a congruence are said to constitute a $(k-1)$-space; the points that satisfy two linearly independent congruences of the type (1) are said to constitute a $(k-2)$-space; and, in general, the points that satisfy $(k-l)$ linearly independent congruences of type (1) are said to constitute an $l$-space. The number of solutions of a set of congruences of type (1) may be counted without much difficulty and the number of points in an $l$-space, $l<k$, found to be $2^{l+1}-1$. In particular, the number of points in a plane (2-space) is seven, and the number in a line (1-space) is three. A single point constitutes a 0 -space. The points common to two $l$-spaces, if there are any, constitute an $r$-space, where $0 \leqq r \leqq l-1$. A set of $l+1$ points which are

* Encyclopédie des Sciences mathématiques, vol. 1, p. 80.

† See Veblen and Bussey, "Finite projective geometries," Transactions Amer. Math. Society, vol. 7 (1906), pp. 241-259. In particular, see \& 2 . 
not all contained in the same $(l-1)$-space is contained in one and only one $l$-space. The $l+1$ points of such a set, if taken $l$ at a time, determine a number of $(l-1)$-spaces whose points constitute a set that may conveniently be called a simplex* of order $l$. The $l+1$ points are called vertices. A convenient symbol for a simplex of order $l$ is $S(l+1)$. Any $i+1$ of the vertices of a $S(l+1)$ are the vertices of a simplex $S(i+1)$ whose points are all contained in the $S(l+1)$.

THEOREM. The number of points in a simplex of order $l$ is one less than the number of points in the l-space determined by its $l+1$ vertices.

By actual count, the theorem is true for $l \leqq 3$. The rest of the proof consists in showing that it can be proved for a simplex $S(m+1)$ if it be assumed true for every simplex $S(l+1)$ for which $l<m$. This is done by arranging the points of the simplex $S(m+1)$ in the $m$ following sets. The sets are not mutually exclusive.

1. The $m+1$ vertices of the simplex $S(m+1)$.

2. The points of the ${ }_{m+1} C_{2}$ lines determined by the vertices taken two at a time.

3. The points of the ${ }_{m+1} C_{3}$ planes determined by the vertices taken three at a time.

4. The points of the ${ }_{m+1} C_{4} 3$-spaces determined by the vertices taken four at a time.

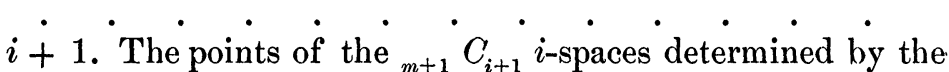
vertices taken $i+1$ at a time.

n. The points of the ${ }_{m+1} C_{m}(m-1)$-spaces determined by the vertices taken $m$ at a time.

[Note: The symbol ${ }_{m+1} C_{j}$ means the number of combinations of $m+1$ things taken $j$ at a time.]

The set numbered $i+1$, being any one of the numbers $1,2,3, \cdots, m$, consists of the points contained in ${ }_{m+1} C_{i+1} i$ spaces each of which is determined by $i+1$ of the vertices of the simplex $S(m+1)$ or, in other words, by the $i+1$ vertices of a simplex $S(i+1)$ which is contained in the simplex $S(m+1)$. By hypothesis, each of these $i$-spaces contains one and only one

* The word is used in geometry of $n$-dimensions to denote the configuration analogous to the triangle in the plane or the tetrahedron in 3-space. 
point not contained in the simplex $S(i+1)$ that determines it. But that one point is a point of the simplex $S(m+1)$ by the very definition of simplex. Therefore, if one begins to count with the first set and counts through the sets in order, the number of points in the set numbered $i+1$ that have not been counted in any previous set is ${ }_{m+1} C_{i+1}$. It follows that the number of points in the simplex $S(m+1)$ is

$$
\sum_{j=1}^{m}{ }_{m+1} C_{j}=2^{m+1}-2
$$

which is one less than the number of points in the $m$-space determined by the $m+1$ vertices of the simplex.

From this theorem it follows that the $l+1$ vertices of a simplex of order $l$ determine uniquely another point, namely, the one point of the $l$-space determined by the simplex that is not also a point of the simplex. It is convenient to call this point the point complementary to the simplex. The triads, tetrads, pentads, etc. of the Steiner problem are found as follows: Every simplex $S(2)$ determines a triad consisting of its two vertices and the complementary point; every simplex $S(3)$ determines a tetrad consisting of its three vertices and the complementary point; and, in general, every simplex $S(l-1)$, $l \leqq k+2$, determines an $l$-ad consisting of the $l-1$ vertices and the complementary point. There are no $l$-ads for $l>k+2$.

When $n=2^{6}-1=63$, it is possible to arrange the $n$ elements in triads, tetrads, pentads, hexads, and heptads. There is no arrangement of the 63 elements in $l$-ads for $l>7$. This special case was involved in Steiner's investigation of the configuration of the 28 double tangents of a quartic curve $*$ and led him to propose for solution the "Combinatorische Aufgabe" which I have called "The tactical problem of Steiner."

\section{ON THE SO-CALLED GYROSTATIC EFFECT.}

BY PROFESSOR ALEXANDER s. CHESSIN.

(Read before the American Mathematical Society, April 24, 1909.)

IN computing the resisting couple of gyrostats or the so-called "gyrostatic effect" it is customary to assume that it is equal to $C \lambda \omega \sin \theta$, where $C, \lambda, \omega$ and $\theta$ denote respectively the moment of inertia of the gyrostat about its geometrical axis, the angular

* Journal für die reine und angewandte Mathematik, vol. 49, pp. 265-272. 